ENCYCLOPEDDE Encyclopédie berbère

BERBERE

4 | 1986

4 | Alger - Amzwar

\title{
Allées couvertes (Kabylie)
}

Voir (Aït) Raouna

G. Camps

\section{OpenEdition}

Journals

Édition électronique

URL : http://journals.openedition.org/encyclopedieberbere/2449

DOI : $10.4000 /$ encyclopedieberbere. 2449

ISSN : 2262-7197

Éditeur

Peeters Publishers

Édition imprimée

Date de publication : 1 septembre 1986

Pagination : 531-533

ISBN : 2-85744-282-3

ISSN : $1015-7344$

Référence électronique

G. Camps, «Allées couvertes (Kabylie)», Encyclopédie berbère [En ligne], 4 | 1986, document A168, mis en ligne le 01 décembre 2012, consulté le 15 octobre 2020. URL : http://journals.openedition.org/ encyclopedieberbere/2449; DOI : https://doi.org/10.4000/encyclopedieberbere.2449

Ce document a été généré automatiquement le 15 octobre 2020

(c) Tous droits réservés 


\title{
Allées couvertes (Kabylie)
}

\author{
Voir (Aït) Raouna
}

\section{G. Camps}

1 Le nord-est de la Kabylie possède un type très original de monument mégalithique qui n'a son équivalent nulle part ailleurs au Maghreb alors que se manifestent certaines affinités entre ces monuments et certains des Iles Baléares et de Sardaigne connus respectivement sous les noms de Navetas et de Tombes des Géants.

2 Il leur a été donné le nom d'allées couvertes (G. Camps, 1959) mais il est préférable de leur adjoindre le qualificatif «kabyles» afin d'éviter toute confusion avec les monuments d'Europe qui sont beaucoup plus anciens bien que d'aspect identique.

3 Les allées couvertes kabyles ne sont connues qu'en deux localités, à Ait Raouna*, sur la côte, à $17 \mathrm{~km}$ à l'est de Tigzirt et à Ibarissen, à $8 \mathrm{~km}$ à l'ouest de Toudja. Ces quatorze monuments (8 à Ait Raouna, 6 à Ibarissen) présentent le même aspect et le même plan : de longs murs parallèles en blocs de grès de fortes dimensions (jusqu'à $3 \mathrm{~m}$ de longueur à Ait Raouna) dessinent une chambre allongée qui ne se différencie du couloir ni dans sa structure ni dans sa largeur qui ne dépasse guère 1,50 m. Les murs s'élèvent bien plus haut que dans les autres monuments mégalithiques nord-africains, la hauteur intérieure du sol à la couverture dépasse toujours $2 \mathrm{~m}$ et atteint même, 3,60 $\mathrm{m}$ dans l'une des allées couvertes d'Ibarissen. La longueur des monuments les mieux conservés varie entre 8 et $15 \mathrm{~m}$. Il n'y a pas d'orientation préférentielle, elle est toujours commandée par la topographie.

4 De telles constructions ne sont pas passées inaperçues : les habitants d'Aït Raouna les appellent les «petites maisons des Romains", tandis qu'une des allées couvertes d'Ibarissen est la " grotte de l'ogre » (Ifri u-wayzen). Dans certains de ces monuments un dépôt votif de microcéramiques confirme leur relation reconnue avec le monde des génies.

5 La couverture de ces allées est assurée, sauf dans un cas, par de grandes dalles dont certaines dépassent $3 \mathrm{~m}$ de longueur, ce qui est exceptionnel dans le mégalithisme nord-africain. Comme la plupart des monuments ont été construits sur des versants et que leur axe correspond à la ligne de plus grande pente les dalles ont été imbriquées en se recouvrant partiellement comme les tuiles d'un toit. A Ibarissen, l'allée couverte 
située dans le village présente une disposition curieuse : les dalles de couverture se superposent faiblement depuis l'entrée jusqu'au milieu de la chambre puis s'abaissent progressivement vers le chevet de sorte que le plafond est plus élevé au centre qu'à l'entrée et au fond. Or la topographie aurait permis ici de placer simplement les dalles côte à côte.

Entrée et intérieur d'une allée couverte d'Ibarissen (photo M. Gast).
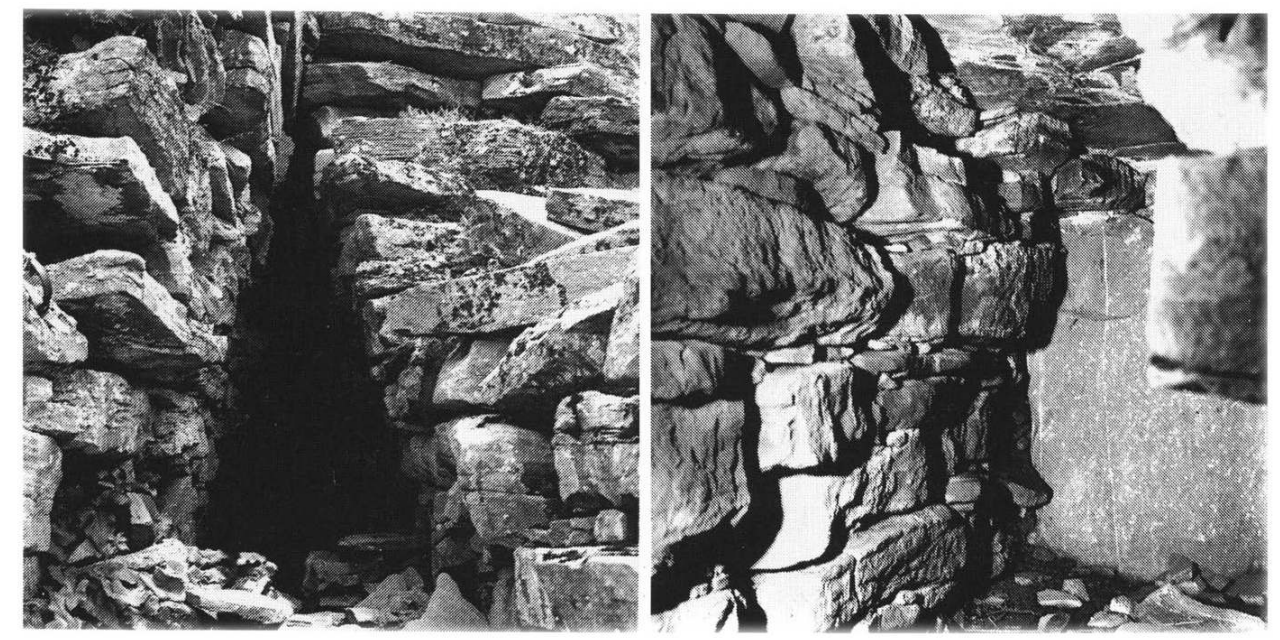

La plus intéressante des allées couvertes d'Ibarissen n'a pas de couverture mégalithique, les deux murs ont été construits avec un contre-fruit considérable puisque distants de $1,10 \mathrm{~m}$ au sol ils sont jointifs au sommet où s'enclavent une suite de parpaings allongés jouant le rôle de clés de voûte. Une telle construction sans encorbellement exige une grande hauteur des murs latéraux pour éviter l'éboulement ; effectivement, la hauteur intérieure est de 3,25 $\mathrm{m}$. Cette même allée présente une autre singularité : un diverticule latéral s'ouvre prependiculairement au couloir, sur la paroi de gauche, à 7,40 $\mathrm{m}$ de l'entrée. Ce diverticule long de $2 \mathrm{~m}$ est recouvert de dalles. Dans d'autres cas le contre-fruit des murs est moindre et la couverture est faite de dalles, la section de la chambre est alors trapézoïdale.

7 Le chevet peut présenter plusieurs aspects; le cas le plus fréquent est celui du faux encorbellement de parpaings qui réduit considérablement la hauteur du fond de la chambre. A Ibarissen on a préféré planter de chant une grande dalle qui est plaquée contre la paroi inférieure du mur de chevet. Dans l'allée située dans le village d'Ibarissen une autre dalle plantée à $0,95 \mathrm{~m}$ en avant de la dalle de chevet détermine une sorte de loge qui pourrait bien être un sarcophage; actuellement, les sédiments amoncelés entre ces deux parois constituent une banquette sur laquelle sont déposées des microcéramiques votives.

8 Implantées le plus souvent sur des versants à forte pente ces allées ont nécessité au préalable le creusement d'une large tranchée dans laquelle fut édifiée au moins la partie la plus éloignée de l'entrée et le chevet, la partie antérieure du monument était à l'extérieur et ce fut celle qui a le plus souffert. Cette disposition donne aux allées couvertes kabyles l'aspect d'entrée de galerie de mine. Il est difficile de dire si la partie extérieure était noyée sous un tumulus; vu l'état actuel il semble que les murs étaient étayés extérieurement par un amas de blocs et de pierraille. 
Allée couverte d'Ibarissen $n^{\circ} 2$ (photo M. Gast).

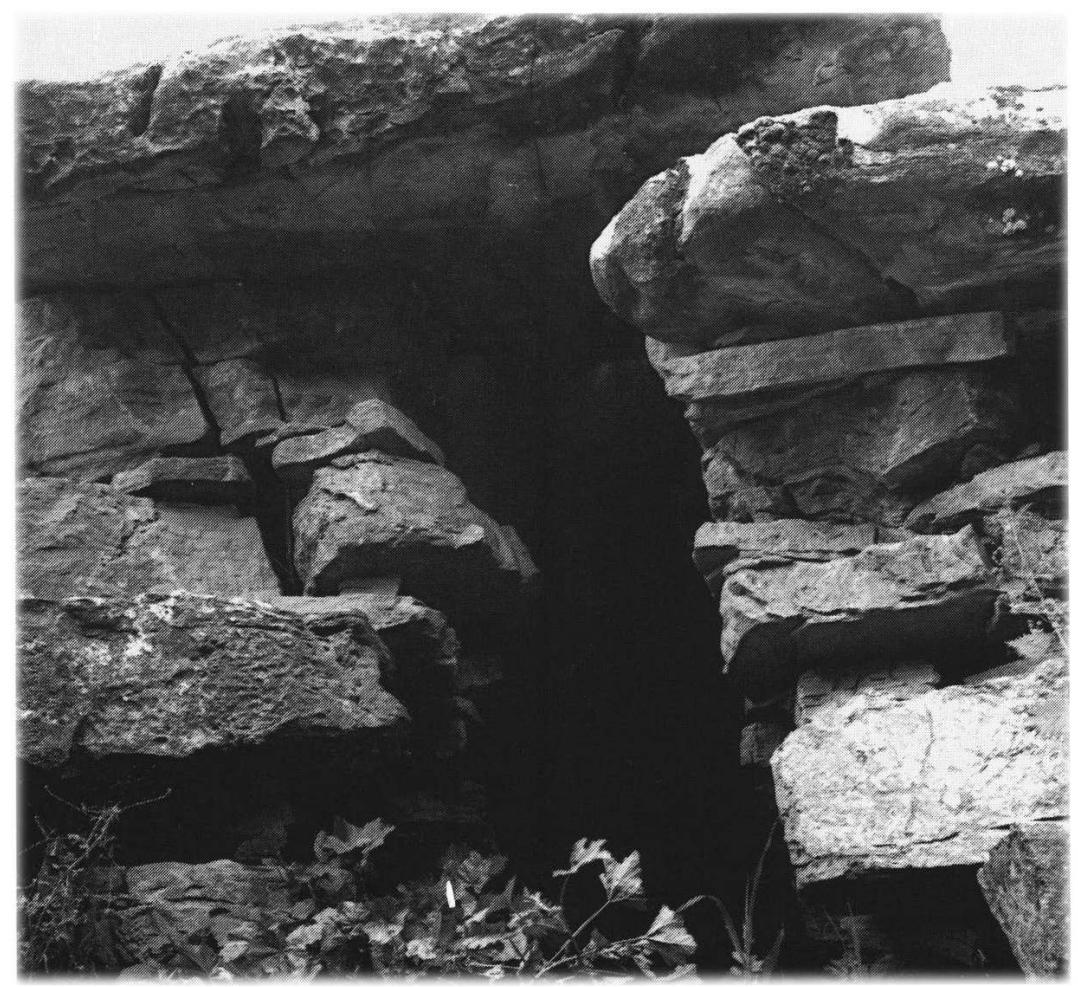

Allée couverte d'Aït Raouna (photo G. Camps).

Image 3.png

Seuls les monuments d'Aït Raouna* ont été fouillés par J.-Cl. et J.-M. Musso. Leurs travaux ont permis de reconnaître, dans deux des monuments les mieux conservés, l'existence d'un caveau aussi large que la chambre mais n'occupant que la moitié la plus profonde de l'allée, sous un dallage intermédiaire encastré de part et d'autre dans les murs latéraux. Des ossements humains sans connexion, rassemblés en tas, étaient accompagnés de céramiques étrusco-campiennes à vernis noir des iv ${ }^{\mathrm{e}}-\mathrm{II}^{\mathrm{e}}$ siècles av. J.-C. Os et poteries se trouvaient aussi bien dans le caveau qu'au-dessus du dallage. Le reste du mobilier comprenait des perles en pâte de verre et des objets en métal (anneaux, clous). L'âge récent de ces monuments, ainsi déterminé par ce mobilier, pose un problème délicat. L'examen de la seule architecture tendrait, en effet, à rapprocher les allées couvertes de Kabylie des navetas des Iles Baléares et des Tombes de Géants de Sardaigne. Les ressemblances ne se limitent pas aux seuls aspects généraux et aux similitudes de dimensions mais s'étendent jusqu'à des aménagements aussi originaux que les galeries ou caveaux superposés qui apparaissent aussi bien dans la Nau d'es Tudons (Minorque) et la Tombe des Géants de Li Lolghi (Arzachena, Sardaigne) que dans les allées couvertes d'Aït Raouna.

Le mobilier trouvé dans les allées couvertes d'Aït Raouna n'étant pas intrusif et ne datant pas une nouvelle occupation des monuments, il faut admettre soit que ce type de monument, si son origine est méditerranéenne comme tout semble l'indiquer, a eu une longévité exceptionnelle au Maghreb, soit que la ressemblance formelle avec les monuments allongés des îles méditerranéennes n'est qu'une simple convergence. Quoi qu'il en soit, leur originalité et même leur spécificité kabyle ne peut être mise en doute. 


\section{BIBLIOGRAPHIE}

Voir (Ait) Raouna.

INDEX

Mots-clés : Protohistoire 